\title{
Angiostrongylus Cantonensis y el caracol africano gigante como causantes de meningitis eosinofilica
}

\author{
Angiostrongylus cantonensis and Giant African Snail which \\ cause Eosinophilic Meningitis
}

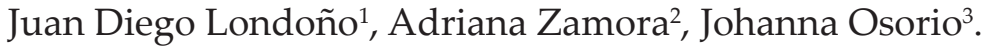

1 Estudiante de medicina. Semillero Investigación en Toxicología (SEITOX)

Grupo de Investigación en Desarrollo Social, Salud Pública y Derechos humanos. Universidad Surcolombiana.

2 Toxicóloga clínica. Directora del Semillero Investigación en Toxicología (SEITOX). Grupo de Investigación en Desarrollo Social, Salud Pública y Derechos humanos. Docente de la Universidad Surcolombiana. Toxicóloga del Hospital Universitario de Neiva.

3 Infectóloga. Docente de la Universidad Surcolombiana. Infectóloga del Hospital Universitario de Neiva. Grupo de Investigación InfectoControl.

Correspondencia: Adriana Zamora, Correo electrónico: adrianazamora73@hotmail.com

\section{Resumen}

El Achatina fulica es reconocido como una plaga que destruye cultivos, igualmente debido a su consumo excesivo de plantas ha llegado a causar alteraciones ambientales, generando un desequilibrio en el ecosistema. Se conoce que en América, A. fulica actúa como hospedero de Angiostrongylus cantonensis, que ocasiona en humanos por infección accidental, meningoencefalitis eosinofílica (ME), angiostrongiliasis abdominal y ocular. La siguiente revisión, se realizó con información proveniente de artículos (nacionales e internacionales) de la biblioteca de PubMed, Ovid, Embase y Medline, entre 1997 y el 2014.

El ser humano corresponde a un hospedero accidental del parasito, en donde el nematodo no concluye su ciclo de vida, y debido a esto muere. Es por lo tanto la respuesta agresiva, que realiza el sistema inmunológico del hospedero (dirigido por los eosinofilos y la inmunoglobulina E) la que genera el cuadro severo de inflamación, y las consiguientes manifestaciones clínicas en el hombre. Finalmente, y aunque se ha llegado a aprender mucho sobre la infección con $A$. cantonensis en la últimas décadas, siguen existiendo varias inquietudes sin resolver, tales como la falta de métodos para su oportuno diagnóstico y el tratamiento eficaz de esta zoonosis.

Palabras clave: caracol gigante africano, meningoencefalitis eosinofilica, eosinofilia, Angiostrongylus cantonensis 


\begin{abstract}
Achatina fulica is known as a plague that destroys crops. Given its excessive plant consumption this plague has caused environmental changes, creating an imbalance in the ecosystem. It is known that in America, A. fulica plays the role of a host for Angiostrongylus cantonensis, which causes eosinophilic meningoencephalitis (ME), abdominal and ocular angiostrongyliasis in humans by accidental infection. The following review was done with information of articles (national and international) from the PubMed, Ovid, Embase y Medline library, between 1997 and 2014.

The human being is an accidental host of the parasite, where the nematode does not end its life cycle, which causes its death. It is therefore, the aggressive response performed by the host's immune system (conducted by eosinophils and immunoglobulin) which brings about severe symptoms of inflammation, and subsequent clinical signs in man. Finally, although in the last decades much has been learnt about the infection with $A$. cantonensis, several concerns remain unresolved, such as the lack of methods to establish an early diagnosis and effective treatment of this zoonosis.
\end{abstract}

Keywords: giant African snail, eosinophilic meningoencephalitis, eosinophilia, Angiostrongylus cantonensis.

\section{Introducción}

El Achatina fulica, igualmente conocido como "caracol gigante africano", es una especie originaria de Africa oriental, desde donde se ha dispersado a diferentes regiones tropicales y subtropicales del mundo (Islas Antillas, Cuba, Brasil y Venezuela), por motivos diversos (cosmética, tráfico de mascotas), convirtiéndose en un problema de índole médico-sanitaria, debido a que este gastrópodo puede actuar como hospedador intermediario del nematodo del género Angiostrongylus, que parasita comúnmente ratas y otros animales, pero que pueden afectar al hombre. ${ }^{(1)}$

Hace 25 años se informó por primera vez en Cuba y en el hemisferio occidental de la aparición de una enfermedad emergente: la ME producida por larvas del nematodo Angiostrongylus cantonensis. Posteriormente esta enfermedad y el parásito que la ocasiona, se han notificado en diversas partes del hemisferio occidental, especialmente el área del caribe y el sur de Estados Unidos. ${ }^{(2)}$

En Colombia se registra la primera aparición del Achatina fulica en la ciudad de Sincelejo, departamento de Sucre, costa caribe de Colombia, en el 2010, luego en el 2011 en los departamentos de Arauca, Boyacá, Caquetá, Casanare,
Guainía, Huila, Meta, Nariño, Putumayo, Santander, Tolima, Valle del Cauca y Vaupés. En 2012 fue localizada en el departamento de Antioquia. $^{(3)}$

De esta manera el presente trabajo pretende recopilar la información y experiencia acumulada sobre esta zoonosis en los últimos años, ya que a pesar de que no se han presentado oficialmente casos en humanos de esta enfermedad en nuestro país, el especialista debe estar preparado para su oportuno diagnóstico y adecuado tratamiento.

\section{Epidemiología}

Considerando al Angiostrongylus cantonensis como un nematodo de gran importancia por su afección a la salud humana, siendo el causante de ME o angiostrongiliasis cerebral, es necesario conocer la distribución de su portador, el Achatina fulica o caracol gigante africano y con ello prevenir la transmisión, que se da por consumo de alimentos contaminados o de caracoles crudos / cocidos.

Achatina fulica es el principal responsable de la propagación del parásito al sudeste de Asia y el Pacífico, el cual alcanzó estas regiones a través de medios humanos con fines comerciales. Habita en zonas con climas tropicales y subtropica- 
les, siendo una especie muy resistente, durante su desarrollo puede alcanzar una longitud de 10 a $30 \mathrm{~cm}$, es fértil a partir de los 5-6 meses de edad, y puede producir de 100 a 200 huevos en el primer año y aumentar su tasa de reproducción (Figura 1). El parásito posee una gran capacidad para infectar a A. fulica, como se muestra en estudios reportados en Brasil y China. ${ }^{(4)}$

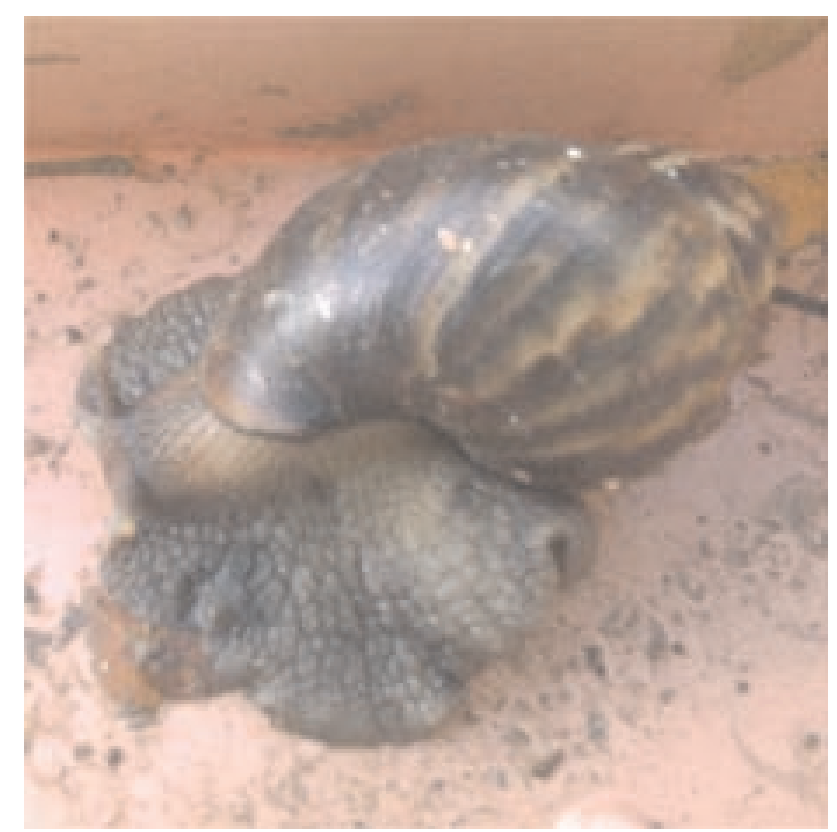

Figura 1: Achatina fulica (Caracol Gigante Africano), especie hermafrodita ovípara, puede alcanzar una longitud de 10 a $30 \mathrm{~cm}$. Fuente: Foto original, cortesía por Felipe Navarro.

Los primeros casos se reportaron desde 1981, en Cuba, sin embargo, ya se había descrito la aparición del $A$. fulica en regiones del Brasil, debido al uso comercial del nematodo en esta zona. La población pediátrica fue la primera afectada, la cual mostro las características clínicas generadas por la infección a causa del parasito, pero no fue hasta después de determinado tiempo que la enfermedad o la evidencia del nematodo que la produce fueran notificadas en países como Puerto Rico (1986), República Dominicana (1992), Jamaica (1996), Haití (2003) y Venezuela (2007). ${ }^{(5)}$
A pesar de que no se han informado de brotes epidémicos en ninguna región de América, si se han reportado brotes en Europa, procedentes de viajeros que regresaban del Caribe. ${ }^{(2,6)} \mathrm{Sin}$ embargo, hay que tener en cuenta que la emergencia de diversos casos aislados en países americanos, es una alerta a las entidades médicas, las cuales deben estar prevenidas para realizar su intervención oportuna. Por otro lado, se ha observado que en Estados Unidos a pesar de no presentarse reportes de pacientes enfermos, sí se ha visto afectada la salud en animales salvajes y domésticos.

En la actualidad, frecuentemente se observan reportes de esta enfermedad en niños, los cuales han estado en contacto con secreciones de moluscos terrestres (babosas o caracoles), en las cuales se encuentran larvas infectivas, igualmente hay situaciones en que son deglutidas, produciéndose una infección masiva del microorganismo, y que ante un inadecuado diagnóstico y tratamiento, pueden ser fatales.

De acuerdo a lo anterior, se considera que hay un inadecuado registro de esta zoonosis que, si bien, aún no ha tenido impacto en la salud pública, sí ha producido fallecimientos, tanto de población adulta como pediátrica. ${ }^{(3)}$

\section{Parasitología}

A. cantonensis corresponde a un helminto, phylum nematodo ${ }^{(7)}$ descrito por primera vez en China (1935), donde se observó que habitaba comúnmente a nivel de las arterias pulmonares de las ratas (Rattus rattus, $R$. norvegicus), y es una de las causas más frecuentes de ME o neuroangiostrongiliasis en el ser humano, cuya infección fue detectada por primera vez en Taiwán (1945). ${ }^{(8)}$

Según su clasificación taxonómica, hacen parte del phylum: nematoda; clase: nematoda; orden: Strongylida; superfamilia: Metastrongyloidea; género: Angiostrongylus; especie: cantonensis. Sin embargo, en la actualidad, varios autores consideran al microorganismo como miembro del 
subgénero Parastrongylus, que en conjunto con otras especies, afectan a roedores de todo el mundo. Todos ellos parasitan a mamíferos, la mayoría habitan en los pulmones de sus hospederos definitivos y, en muy pocas excepciones, sus hospederos intermediarios son gasterópodos. ${ }^{(2)}$

Se han descrito alrededor de 20 especies del género Angiostrongylus, las cuales se han encontrado en roedores, carnívoros e insectívoros en todo el mundo; pero sólo dos de ellos (A. cantonensis y $A$. costaricensis) afectan al hombre. A. cantonensis, causa alteraciones en el sistema nervioso central (SNC) y en ciertas ocasiones a nivel pulmonar, por otro lado $A$. costaricensis, habita las arterias mesentéricas, generando angiostrongiliasis abdominal, conocida en América tropical. ${ }^{(2)}$
Se han encontrado otras especies, con ciclos similares al de $A$. cantonensis (A. malaysiensis y $A$. mackerrasae) sin embargo, no se han reportado casos de infección en humanos. Según estudios realizados de manera experimental en monos, se ha reportado que $A$. malaysiensis puede producir alteraciones neurológicas en estos seres. ${ }^{(2)}$

\section{Ciclo de Vida}

Su ciclo de vida es complejo; los nematodos adultos necesitan de un hospedero definitivo el cual habitar, es común encontrarlos en las arterias pulmonares de ratas y otros roedores, e igualmente necesitan de un huésped intermediario (molusco) el cual es el más apropiado, comúnmente caracoles o babosas. A. cantonensis se encuentra en el medio exterior en forma de larvas L1, posteriormente llegan a su hospedero

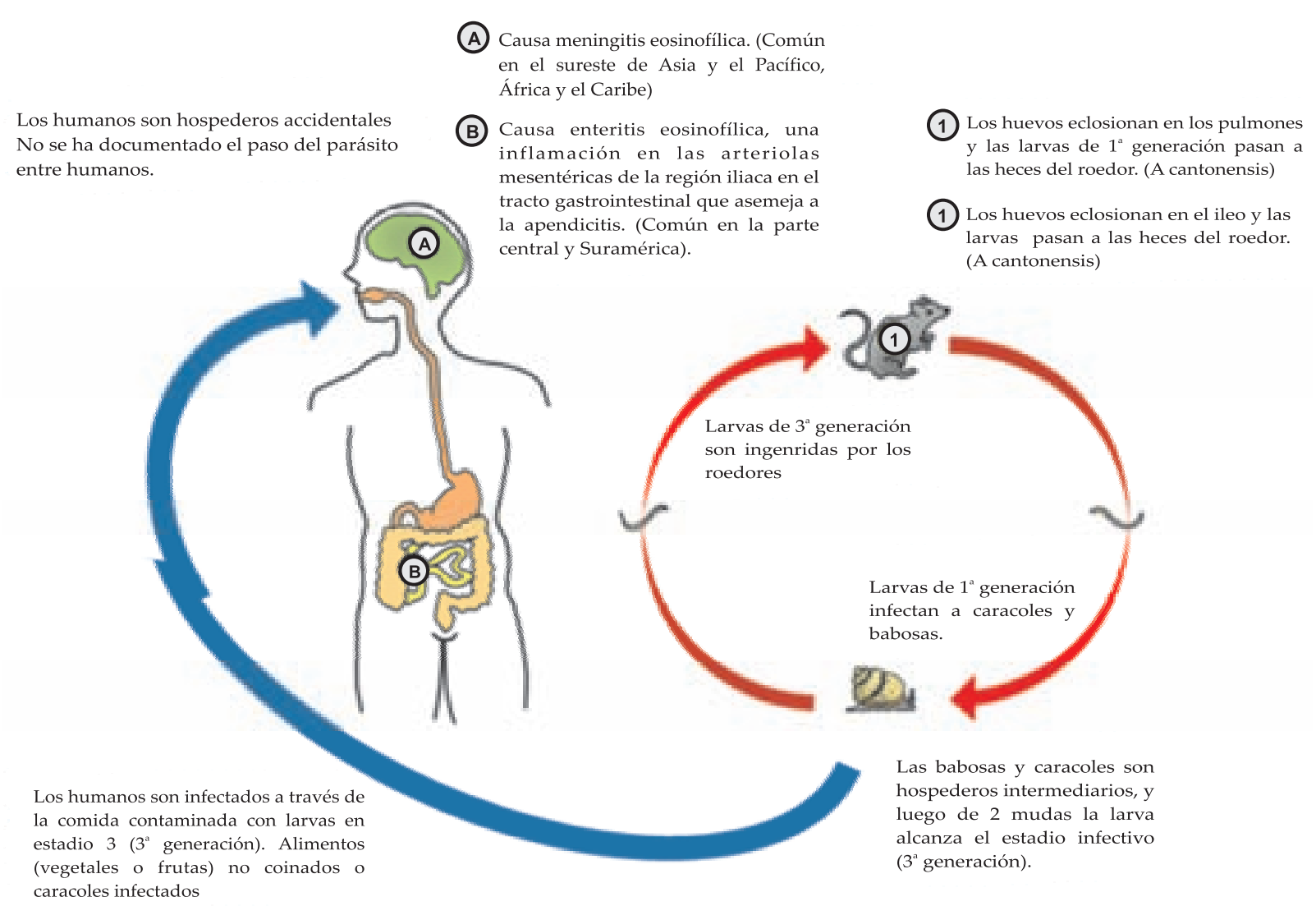

Figura 2: Ciclo de vida en el ratón (hospedero definitivo), Angiostrongylus cantonensis, lo que causa meningitis eosinofílica, Angiostrongylus costaricensis, que causa enteritis eosinofílica. Fuente: modificación de imagen CDC, disponible en http://www.dpd.cdc.gov/dpdx. 
intermediario (caracoles o babosas) a través de la ingesta de componentes de su hábitat. Dentro del caracol sufren dos mudas larvarias, hasta llegar a convertirse en larvas de tercer estadio (L3), que resultan infectivas para los hospederos definitivos (mamíferos). Al ingerir el molusco o sus secreciones, las larvas L3 migran al cerebro del mamífero, donde van a sufrir dos mudas larvarias más, hasta llegar a convertirse en larvas de quinto estadio (L5) o adultos jóvenes (en 4 semanas). ${ }^{(2)}$

Las formas adultas del parásito maduran en el cerebro de las ratas, posteriormente entran en la circulación central, y se aparean en las arterias pulmonares, donde depositan sus huevos. Éstos se convierten en larvas de primera generación L1, las cuales penetran en los vasos pulmonares para acceder al árbol respiratorio, donde son expulsadas por el hospedero al toser, o son deglutidas y posteriormente excretadas en las heces. Las larvas de segunda generación L2 deben ser consumidas por caracoles de tierra (como Achatina fulica) o babosas, con el fin de madurar y convertirse en larvas de tercera generación L3, que sirven de alimento para los roedores y con ello mantienen su ciclo de vida. (Figura 2)

El hombre se convierte en un hospedero accidental, al consumir moluscos (huéspedes intermediarios), alimentos (frutas, verduras sin lavar) contaminados con larvas de caracoles o babosas, o al consumir pescado crudo, crustáceos (camarones, cangrejos) o anfibios (ranas) que consumen los moluscos infectados. En el hombre las larvas migran al SNC y tratan de alcanzar su madurez sexual, sin embargo y con el tiempo las larvas no llegan a este estadio de madurez, por lo tanto mueren y causan la ME.

\section{Manifestaciones Clínicas}

La mayoría de pacientes que cursan con la infección manifiestan cefalea pulsátil, sin alteración de la conciencia, asociada a otros síntomas como fiebre, parestesias, debilidad muscular, dolor orbital o retro-orbital, diplopía, ataxia, náuseas, vómitos, dolor abdominal y erupciones en la piel. Al realizar el examen físico se pueden apreciar rigidez de cuello, máculas corneales, brudzinski, hiperestesias, parálisis de VI y VII par e insuficiencia laberíntica transitoria. El cuadro clínico se resuelve espontáneamente, sin secuelas neurológicas graves, debido a que las larvas no sobreviven en huéspedes humanos. ${ }^{(9)}$ Se han descrito casos de pacientes que cursan con angiostrongylosis ocular, con presencia de disminución de la agudeza visual y diplopía, encontrando hallazgos en el fondo del ojo como alteración del epitelio pigmentario de la retina con pliegues en su superficie. De igual manera reportan el hallazgo de larvas estadio L5 vivas a nivel del vítreo. ${ }^{(10)}$

\section{Angiostrongilosis Abdominal}

La angiostrongilosis abdominal es debido en su mayoría a otras especies de Angiostrongylus como el $A$. costaricensis, sin embargo también puede ser causada por $A$. cantonensis. ${ }^{(11)}$ Este cuadro se caracteriza por trombosis en los vasos que irrigan el intestino y otros órganos abdominales, ocasionando apendicitis o la inflamación y en muchos casos la necrosis de dichos tejidos. (1)

\section{Meningitis Eosinofílica o Angiostrongiliasis Cerebral}

Esta patología es rara en los países occidentales, pero en ocasiones se observa en asociación a otras enfermedades como la tuberculosis, sífilis y coccidioidomicosis. Igualmente existen otras infecciones por helmintos que pueden causar un cuadro similar de ME, entre ellos la cisticercosis, esquistosomiasis, paragonimiasis y equinococosis. La angiostrongiliasis cerebral es más común en las regiones Pacífico y Sur Oriental de Asia, trasmitida por A. cantonensis..$^{(9,12)}$

Además de $A$. cantonensis se tiene conocimiento de otras dos especies de parásitos nematodos, que causan un cuadro de ME, y cuyas manifestaciones pueden ser más severas, ellos son el $B a-$ ylisascaris procyonis y Gnanthostoma spinigerum. 
Gnanthostoma, causa una forma particularmente severa de mieloencefalitis eosinofílica que se manifiesta en dolor neuropático, monoplejia, y hemorragia subaracnoidea asociada a edema cerebral, erupciones cutáneas y larvas migrans a nivel visceral y cutáneo. La expresión aumentada de la enfermedad parece ser el resultado de una vigorosa respuesta inmune del huésped a las larvas en el SNC. Es posible hacer un diagnóstico diferencial de estas infecciones a través de pruebas serológicas. ${ }^{(8,13,14)}$ Según un estudio realizado en Taiwán a partir de 31 pacientes con meningitis eosinofílica y manifestaciones clínicas sugestivas del cuadro característico causado por el nematodo, se encontró anticuerpos plasmáticos positivos en $84 \%$ para A. cantonensis. ${ }^{(15)}$

La mayoría de los casos de angiostrongiliasis cerebral han sido reportados en niños expuestos al caracol gigante africano (A. fulica), sin embargo, también se han evidenciado este tipo de manifestaciones entre un rango de edad de 2 hasta los 80 años de edad. Estos cuadros estaban en relación con algún antecedente de ingesta o el contacto físico con caracoles o babosas. La enfermedad es mucho más grave en la población pediátrica, debido a una carga más alta de parásitos, en relación con la superficie corporal.(15)

\section{Datos de Laboratorio}

En cuanto a los datos de laboratorio, el cuadro hemático de estos pacientes puede reflejar ciertas características. La mayoría de los pacientes cursan con un recuento de glóbulos blancos normales o ligeramente elevados y con eosinofilia.

Por otro lado el líquido cefalorraquídeo (LCR) se puede encontrar turbio, con un recuento de leucocitos elevados y eosinofilia (50\%), una concentración de glucosa mayor a $40 \mathrm{mg} / \mathrm{dl}$, una elevada cifra de proteínas $(100 \mathrm{mg} / \mathrm{dl})$ y en ocasiones estadios de III generación de larvas en LCR. Las tinciones y cultivos no aportan datos significativos pero son útiles para establecer diagnóstico diferencial de la meningitis.
Anticuerpos contra $A$. cantonensis pueden ser detectados en el suero de los pacientes y en el LCR, sin embargo, esta prueba no está implementada en Colombia. Igualmente, se pueden encontrar elevados niveles de IgE, creatin quinasa, alanina transferasa, deshidrogenasa $y$ amino lactato. ${ }^{(9,16)}$

\section{Eosinofilia}

Se ha informado la presencia de eosinofilia en el $50 \%$ de los casos, la cual tiende a desaparecer dentro de las 3 a 4 semanas siguientes. La respuesta exagerada desencadenada por $A$. cantonensis, en el hospedero accidental, es la causa principal de la severidad del cuadro, y no la presencia del microorganismo en el SNC.

Esta respuesta se basa principalmente en la producción de IgE específica, la cual es capaz de eliminar las larvas de Angiostrongylus con ayuda de las células fágicas (principalmente los eosinófilos), estos descargan su contenido (neurotoxina) siendo ésta la principal responsable de los daños en el ser humano.

Los huéspedes accidentales cursan con un síndrome neurotóxico (ataxia, temblor, parálisis y pérdida de peso) y a nivel histológico, se observa una pérdida o alteración de las células de purkinje a nivel del cerebelo.

La eosinofilia depende de la concentración de parásitos en el huésped accidental y no en los huéspedes definitivos. Por lo tanto y según estudios in vivo, la respuesta es mayor en el huésped accidental y casi nulo en los roedores (huésped definitivo). ${ }^{(2)}$

\section{Inmunoglobulina E (IgE)}

La inmunoglobulina E (IgE), presenta una capacidad citotrópica, la cual le permite un aumento de su vida media a nivel sérico, y que posteriormente se incremente a los tres o cuatro meses, al unirse a receptores específicos en los mastocitos y los basófilos. Dichas células intervienen en procesos alérgicos, por lo que muchos piensan 
que ésta es su función fundamental. No obstante, encontramos receptores para IgE en los macrófagos, los eosinófilos y las plaquetas, lo cual podría explicar su participación en las defensas contra helmintos, particularmente el nematodo A. cantonensis.

Es común detectar niveles de IgE elevados en suero, en las infecciones por helmintos. No se ha determinado si el aumento de los niveles séricos de esta es debido a una función protectora o es simplemente una respuesta a los antígenos extraños. Los niveles de IgE pueden persistir elevados durante largos periodos posterior a la resolución de la infección, (hasta 6 a 18 meses después de la eliminación de los parásitos). En los brotes actuales con $A$. cantonensis, el nivel de esta inmunoglobulina no se asocia con la severidad del cuadro clínico. ${ }^{(9)}$

\section{Líquido Cefalorraquídeo (LCR)}

En el estudio del LCR rara vez se observan larvas, sin embargo al permanecer el paciente en posición sentada durante 1 hora antes de extraer la muestra, aumenta las posibilidades de este hallazgo. ${ }^{(9)}$

El primer diagnóstico, el cual se debe pensar ante un LCR con presencia de eosinofilos y un paciente con alteraciones neurológicas, en países endémicos de $A$. cantonenesis, como lo es Cuba, es en una angiostrongiliasis. ${ }^{(2)}$ Conociendo la presencia del intermediario en Colombia, también se debe sospechar un cuadro de angiostrongiliasis en un paciente con las características anteriores, además de una infección por Gnathostomiasis, explorando la historia epidemiológica.

\section{Imágenes Diagnósticas}

En cuanto al uso de imágenes diagnósticas, se ha reportado el uso de resonancia magnética en pacientes con ME por A. cantonenis que a menudo revelan múltiples engrosamientos nodulares en el tejido cerebral y engrosamientos lineales en las leptomeninges. ${ }^{(10)}$ De igual manera se han encontrado otro tipo de hallazgos en tomografía cerebral como son edema, hiperdensidad leptomeningea, infarto antiguo y dilatación ventricular. En la radiografía de tórax se ha observado engrosamiento pleural apical, infiltrados reticulonodulares difusos y en la ecografía abdominal cambios como hígado graso, hemangioma hepático, masas hepáticas, carcinoma hepatocelular, los cuales aunque no son específicos se han descrito en relación con la ME. ${ }^{(15)}$

\section{Tratamiento}

En la infección del SNC por el A. cantonensis, no se llega a la cronicidad porque las larvas L3 mueren en el LCR, y en caso de que la ingestión de larvas no haya sido masiva, el paciente se recupera con tratamiento sintomático, como en una meningoencefalitis producida por enterovirus. $^{(16)}$

El papel del tratamiento con agentes antihelmínticos y corticoides es controvertido. ${ }^{(17,18,19)}$ No se ha encontrado ninguna diferencia en la duración o gravedad de la enfermedad en los pacientes tratados con analgésicos solos, con analgésicos y glucocorticosteroides, o con analgésicos en asociación con antibióticos. ${ }^{(20,21)}$ Se han reportado buenos resultados con el uso de albendazol o levamisol en estudios no controlados en niños. ${ }^{(22)}$ Por otro lado, el uso de prednisolona por dos semanas, ayuda a disminuir la cefalea y la necesidad de punciones lumbares repetidas. En los casos en que se evidencie manifestaciones oculares como retinitis o neuritis óptica ciertos autores han sugerido el uso de esteroides como tratamiento, sin embargo, otros mencionan un mejor resultado con cirugía o terapia laser. ${ }^{(10)}$

\section{Conclusiones}

El caracol gigante ( $A$. fulica) es considerado ya como una plaga en varias regiones de Colombia y el mundo, además de causar un desequilibrio ambiental, también es el transmisor de A. Cantonensis, un nematodo capaz de causar un cua- 
dro infeccioso, que si no se trata adecuadamente puede ser tan severo que puede ocasionar la muerte. Es necesario por lo tanto, que los países del área donde se han comunicado casos o se ha descrito la presencia del intermediario estén alerta a la propagación de la enfermedad, especialmente en casos de meningitis eosinofílica.

Los seres humanos son hospederos accidentales, en donde ocurre la infestación del parasito, sin embargo este es incapaz de completar su ciclo de vida. La respuesta exagerada por el hospedero es la causante de una enfermedad inmune, la cual se observa con el aumento de los eosinófilos y la IgE.

Es necesario implementar medidas sanitarias que incluyan el control de este intermediario, higiene de manos y adecuada manipulación de alimentos.

A pesar de lo aprendido en las últimas décadas sobre la infección con $A$. cantonensis tanto en América como en el mundo, hace falta implementar métodos que permitan un diagnóstico adecuado en los casos de meningitis eosinofílica, así como determinar las opciones terapéuticas más efectivas.

\section{Bibliografía}

1. MARTÍNEZ-ESCARBASSIERE Rafael, O. MARTÍNEZ Enrique, CASTILLO Otto. Distribución geográfica de Achatina (Lissachatina) fulica (Bowdich, 1882) (Gastropoda-Stylommatophora- Achatinidae) en Venezuela. Memoria de la Fundación La Salle de Ciencias Naturales (2008). 169: 93-106.

2. DORTA-CONTRERAS A.J., NÚÑEZ-FERNÁNDEZ F.A., SÁNCHEZ-ZULUETA E. Peculiaridades de la meningoencefalitis por Angiostrongylus cantonensis en América. [REV NEUROL 2007; 45: 755-63].

3. DE LA OSSA-LACAYO Alejandro, DE LA OSSA V. Jaime, LASSO A. Carlos. Registro del caracol africano gigante Achatina fulica (Bowdich 1822) (Mollusca: Gastropoda-Achatinidae) en Sincelejo, costa Caribe de Colombia. [En Línea]. Biota
Colombiana 13 (2) 2012. [consultado el 6 Ago de 2013].

4. FONTANILLA I.K.C., WADE, C.M. First report of Angiostrongylus cantonensis in the Giant African Land Snail Achatina fulica in French Polynesia detected using the SSU rRNA gene. Tropical Biomedicine 29(4): 642-645 (2012)

5. CALDEIRA Roberta Lima. Carvalho, Omar dos Santos. First record of molluscs naturally infected with Angiostrongylus cantonensis (Chen, 1935) (Nematoda: Metastrongylidae) in Brazil. Mem Inst Oswaldo Cruz, Rio de Janeiro, Vol. 102(7): 887-889, November 2007.

6. GODAN D. Pests slugs and snails. Biology and control. 1983. Berlin, Springer-Verlag, Berlin, 445 pp.

7. PILSCZEK, F. Helminthic infections mimicking malignancy: a review of published case reports. The Journal of Infection in Developing Countries, North America, 4, apr. 2010. Available at: $<$ http://www.jidc.org/index.php/journal/article/ view/20818089/411>. Date accessed: 14 May. 2014

8. DIAZ James H. Recently Reemerging Helminthic Infections Causing Eosinophilic Meningoencephalitis: Neuroangiostrongyliasis, Baylisascariasis, and Gnathostomiasis. Journal of Neuroparasitology. Vol. 1 (2010).

9. HUNG-CHIN Tsai, YUNG-CHING Liu, CHUAN-MIN Yen. Eosinophilic Meningitis Caused by Angiostrongylus Cantonensis: Report of 17 Cases. THE AMERICAN JOURNAL OF MEDICINEt. Volume 111 (2001).

10. SOLÓRZANO Álava, Martini Robles, Hernández Álvarez, Sarracent Pérez, Muzzio Aroca, Rojas Rivero. Angiostrongylus Cantonensis: Un Parásito Emergente En Ecuador. Revista Cubana de Medicina Tropical, Norteamérica, 66, feb. 2014. Disponible en: <http://www.revmedtropical.sld.cu/index.php/medtropical/article/ view/4/3>. Fecha de acceso: 14 may. 2014.

11. MOLINA-DURAN Alexánder. Angiostrongiliasis: Reporte de un caso. Rev. costarric. cienc. méd, San José, v. 27, n. 3-4, dic. 2006. Disponible en $<$ http://www.scielo.sa.cr/scielo.php?script=sci arttext\&pid=S0253-29482006000200007\&lng=es \&nrm=iso>. accedido en 20 agosto 2013. 
12. MILLER Michael A, Menowsky Michael, Leeson Kimberly, Leeson Ben, Eosinophilic meningitis: what's the "diff"? The American Journal of Emergency Medicine. Elsevier, volumen 32, issue 1, january 2014, pages 107.e5-107.e7

13. KANLAYANEE Sawanyawisuth1, Kittisak Sawanyawisuth, Pewpan M Intapan, Piyarat Khotsri, Jaturat Kanpittaya, Verajit Chotmongkol, Wanchai Maleewong. Specificity of immunoblotting analyses in eosinophilic meningitis. Mem Inst Oswaldo Cruz, Rio de Janeiro, Vol. 106(5): 570-572, August 2011

14. MARTIN-ALONSO A, Foronda P, Quispe-Ricalde MA, Feliu C, Valladares B (2011) Seroprevalence of Angiostrongylus cantonensis in Wild Rodents from the Canary Islands. PLoS ONE 6(11): e27747. doi:10.1371/journal.pone.0027747

15. YU-TING Tseng, Hung-Chin Tsai, Cheng Len Sy, Susan Shin-Jung Lee, Shue-Ren Wann, YungHsing Wang, Jei-Kuang Chen, Kuan-Sheng Wu, Yao-Shen Chen. Clinical manifestations of eosinophilic meningitis caused by Angiostrongylus cantonensis: 18 years' experience in a medical center in southern Taiwan. Journal of Microbiology, Immunology and Infection (2011) 44, 382e389

16. RAMIREZ-AVILA Lynn, Sally Slome, Frederick L. Schuster, Shilpa Gavali, Peter M. Schantz, James Sejvar, and Carol A. Glaser. Eosinophilic
Meningitis due to Angiostrongylus and Gnathostoma Species. Emerging infections. Clinical Infectious Diseases Boston, 1 february, 2009; 48:322-7

17. PUNYAGUPTA $S$, Juttijudata P, Bunnag T. Eosinophilic meningitis in Thailand: clinical studies of 484 typical cases probably caused by Angiostrongylus. Am J Trop Med Hyg. 1975(24):921-931.

18. SAWANYAWISUTH Kittisak, Chotmongkol Verajit. Chapter 15. Eosinophilic meningitis. Neuroparasitology and Tropical Neurology, Handbook of Clinical Neurology 2013, Vol.114:207-215.

19. OEHLER E., Ghawche F., Delattre A., Berberian A., Levy M., Valour F. Angiostrongylus cantonensis eosinophilic meningitis: A clinical study of 42 consecutive cases in French Polynesia. Parasitology International 2014 63:3(544-549).

20. HWANG KP, Chen ER. Clinical studies on angiostrongyliasis cantonensis among children in Taiwan. Southeast Asian J Trop Med Pub Health. 1991;22(Suppl):194-199.

21. HWANG KP, Chen ER, Chen TS. Eosinophilic meningitis and meningoencephalitis in children. Acta Paeditr Sinica. 1994;35:124-135.

22. CHOTMONGKOL V, Sawanyawisuth K, Thavornpitak Y. Corticosteroid treatment of eosinophilic meningitis. Clin Infect Dis. 2000;31: 660-662 